\title{
RELATIVE AND TATE COHOMOLOGY OF DING MODULES AND COMPLEXES
}

\author{
Chunxia Zhang
}

\begin{abstract}
We investigate the relative and Tate cohomology theories with respect to Ding modules and complexes, consider their relations with classical and Gorenstein cohomology theories. As an application, the Avramov-Martsinkovsky type exact sequence of Ding modules is obtained.
\end{abstract}

\section{Introduction}

The subject of relative and Tate cohomology theories goes back to Avramov and Martsinkovsky [2]. They studied the theories in the subcategory of modules of finite $G$-dimension and made an intensive study of the interaction between the three cohomology theories: i.e., the absolute, the relative and the Tate cohomology theories.

On the other hand $G$-dimension 0 modules are called Gorenstein projective by Enochs and Jenda [4]. Note that Ding et al. in [3] and [11] considered two special cases of Gorenstein projective and Gorenstein injective modules, which they called strongly Gorenstein flat and Gorenstein FP-injective modules, respectively. Since over a Ding-Chen ring the strongly Gorenstein flat and Gorenstein FP-injective modules have many nice properties analogous to Gorenstein projective and Gorenstein injective modules over a Gorenstein ring, Gillespie [6] renamed these modules as Ding projective and Ding injective modules, respectively.

Subsequent work of the relative cohomology of Ding homological modules has been made by Yang in [17]. He showed that over a Ding-Chen ring $R$, for any $R$-modules $M, N$ and any $i \geq 1$ one has

$$
\operatorname{Ext}_{\mathcal{D P}}^{i}(M, N) \cong \operatorname{Ext}_{\mathcal{D} \mathcal{I}}^{i}(M, N) \text {. }
$$

Though we don't know whether or not every $R$-module has finite Ding injective or Ding projective dimension when $R$ is a Ding-Chen ring (see [3, Thm. 3.6]

Received October 4, 2014; Revised April 15, 2015.

2010 Mathematics Subject Classification. 16E40, 18G10, 18G25.

Key words and phrases. Ding projective (injective) module, relative cohomology, Tate cohomology, Avramov-Martsinkovsky type exact sequence. 
and [11, Lem. 3.1] for partial answer), we have the following interesting result (see Theorem 3.5).

Theorem A. Let $M$ be an $R$-module with finite Ding projective dimension and $N$ be an $R$-module with finite Ding injective dimension. Then for all $i \geq 1$, we have isomorphisms

$$
\operatorname{Ext}_{\mathcal{D} \mathcal{P}}^{i}(M, N) \cong \operatorname{Ext}_{\mathcal{D} \mathcal{I}}^{i}(M, N) .
$$

Let $M$ be an $R$-module admitting a proper left $\mathcal{D} \mathcal{P}$-resolution and $N$ be an arbitrary $R$-module. Recently, Ren, Liu and Yang in [13] considered Iacob's generalized Tate cohomology $\widehat{\operatorname{Ext}}_{\mathcal{D P}}^{n}(M, N)$. Since every $R$-module with finite Ding projective dimension has a proper left $\mathcal{D} \mathcal{P}$-resolution (see Proposition $3.2)$, we define the Tate cohomology $\widehat{\operatorname{Dext}}_{R}^{n}(M, N)$ for an $R$-module $M$ with finite Ding projective dimension and an arbitrary $R$-module $N$. Also we will show that the Tate cohomology of ours and the generalized Tate cohomology of Ren et al.'s are identical under the following general condition (see Theorem $5.2)$.

Theorem B. Let $M$ be an R-module with finite Ding projective dimension. Then for each $R$-module $N$ and each $n \geq 1$ we have

$$
\widehat{\operatorname{Dext}}_{R}^{n}(M, N) \cong \widehat{\operatorname{Ext}}_{\mathcal{D P}}^{n}(M, N) \text {. }
$$

The close relations between absolute, relative and Tate cohomologies are illuminated by an Avramov-Martsinkovsky type exact sequence (see [2, Thm. 7.1]). As an application of the relative and Tate cohomology theories of Ding homological modules, we get the Avramov-Martsinkovsky type exact sequence of Ding homological modules (see Theorem 5.6).

Theorem C. Let $R$ be a Ding-Chen ring. Then for any $R$-modules $M$ with finite Ding projective dimension and $N$ with finite Ding injective dimension there is an Avramov-Martsinkovsky type exact sequence

$$
\begin{aligned}
0 \longrightarrow \operatorname{Ext}_{\mathcal{D I}}^{1}(M, N) \longrightarrow \operatorname{Ext}_{R}^{1}(M, N) \longrightarrow \widehat{\operatorname{Dext}}_{R}^{1}(M, N) \longrightarrow \cdots \\
\longrightarrow \operatorname{Ext}_{\mathcal{D I}}^{i}(M, N) \longrightarrow \operatorname{Ext}_{R}^{i}(M, N) \longrightarrow \widehat{\operatorname{Dext}}_{R}^{i}(M, N) \longrightarrow \cdots
\end{aligned}
$$

The layout of this paper is as follows: In Section 2, we review some definitions and notations which are basic to the rest of the paper. Section 3 is devoted to defining and studying the relative cohomology theory of Ding homological modules. We show that this theory has intimate connections with the classical cohomolgy and Gorenstein cohomology theories. In Section 4, the Tate cohomology theory of complexes with finite Ding injective dimension is treated. Finally, as an application, the Avramov-Martsinkovsky type exact sequence with respect to Ding homological modules is obtained in Section 5. 


\section{Preliminaries and notions}

Throughout this paper, $R$ denotes an associative ring with identity, and modules are left $R$-module. In this section, we recall basic definitions and notions used in this paper.

\subsection{Complex}

The category of left $R$-modules is denoted $\mathcal{M}=\mathcal{M}(R)$ and $\mathcal{C}=\mathcal{C}(R)$ denotes the category of $R$-complexes.

Definition 2.1. An $R$-complex $X$ is a sequence of $R$-modules $X_{l}$ and $R$-linear maps $\partial_{l}^{X}, l \in \mathbb{Z}$

$$
X=\cdots \longrightarrow X_{l+1} \stackrel{\partial_{l+1}^{X}}{\longrightarrow} X_{l} \stackrel{\partial_{l}^{X}}{\longrightarrow} X_{l-1} \longrightarrow \cdots
$$

such that $\partial_{l}^{X} \partial_{l+1}^{X}=0$ for all $l \in \mathbb{Z}$. For an $R$-complex $X$ and any $i \in \mathbb{Z}$, let $Z_{i}(X)=\operatorname{Ker}_{i}^{X}, B_{i}(X)=\operatorname{Im} \partial_{i+1}^{X}$, and $C_{i}(X)=\operatorname{Coker}_{i+1}^{X}$. The residue class module $\mathrm{H}_{i}(X)=Z_{i}(X) / B_{i}(X)$ is called the $i$ th homology module of $X$. The homology complex $\mathrm{H}(X)$ is defined by setting $\mathrm{H}(X)_{i}=\mathrm{H}_{i}(X)$ and $\partial_{i}^{\mathrm{H}(X)}=0$ for all $i \in \mathbb{Z}$. Furthermore, we set $\sup X=\sup \left\{i \in \mathbb{Z} \mid X_{i} \neq 0\right\}$ and $\inf X=\inf \left\{i \in \mathbb{Z} \mid X_{i} \neq 0\right\}$.

Given an $R$-module $M$, we denote by $\underline{M}$ the complex with $M$ in the 0 th place and 0 elsewhere, and identify $M$ with $\underline{M}$ occasionally if there is no risk of ambiguity.

Definition 2.2. Given an $R$-complex $X$ and an integer $n, \Sigma^{n} X$ denotes the complex $X$ shifted $n$ degrees to the left, i.e., $\left(\Sigma^{n} X\right)_{i}=X_{i-n}$ and $\partial_{i}^{\Sigma^{n} X}=$ $(-1)^{n} \partial_{i-n}^{X}$.

Definition 2.3. A homomorphism $\varphi: X \rightarrow Y$ of degree $n$ is a family $\left(\varphi_{i}\right)_{i \in \mathbb{Z}}$ of homomorphisms of $R$-modules $\varphi_{i}: X_{i} \rightarrow Y_{i+n}$. All such homomorphisms form an abelian group, denoted $\operatorname{Hom}_{R}(X, Y)_{n}$; it is clearly isomorphic to $\Pi_{i \in \mathbb{Z}} \operatorname{Hom}_{R}\left(X_{i}, Y_{i+n}\right)$. We let $\operatorname{Hom}_{R}(X, Y)$ denote the complex of $\mathbb{Z}$-modules

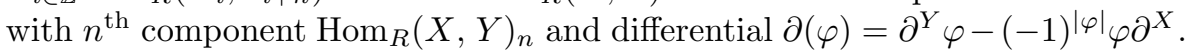
if

A homomorphism $\varphi \in \operatorname{Hom}_{R}(X, Y)_{n}$ is called a chain map if $\partial(\varphi)=0$, i.e.,

$$
\partial_{i+n}^{Y} \varphi_{i}=(-1)^{|\varphi|} \varphi_{i-1} \partial_{i}^{X} \text { for all } i \in \mathbb{Z}
$$

A chain map of degree 0 is called a morphism. Homomorphisms $\varphi$ and $\varphi^{\prime}$ in $\operatorname{Hom}_{R}(X, Y)_{n}$ are called homotopic, denoted $\varphi \sim \varphi^{\prime}$, if there exists a degree $n+1$ homomorphism $\mu$, called a homotopy, such that $\partial(\mu)=\varphi-\varphi^{\prime}$. A homotopy equivalence is a morphism $\varphi: X \rightarrow Y$ for which there exists a morphism $\psi: Y \rightarrow X$ such that $\varphi \psi \sim i d^{Y}$ and $\psi \varphi \sim i d^{X}$.

Definition 2.4. A morphism $\alpha: X \rightarrow Y$ of $R$-complexes is called a quasiisomorphism if $\mathrm{H}(\alpha)$ is an isomorphism. For a morphism $\alpha: X \rightarrow Y$, we denote by $\operatorname{Cone}(\alpha)$ the mapping cone of $\alpha$. It is given by Cone $(\alpha)_{i}=Y_{i} \oplus X_{i-1}$ and $\partial_{i}^{\text {Cone }(\alpha)}\left(y_{i}, x_{i-1}\right)=\left(\partial_{i}^{Y}\left(y_{i}\right)+\alpha_{i-1}\left(x_{i-1}\right),-\partial_{i-1}^{X}\left(x_{i-1}\right)\right)$. 


\subsection{Ding homological modules}

We denote the classes of projective, flat, injective and FP-injective $R$-modules by $\mathcal{P}=\mathcal{P}(R), \mathcal{F}=\mathcal{F}(R), \mathcal{I}=\mathcal{I}(R)$ and $\mathcal{F} \mathcal{I}=\mathcal{F} \mathcal{I}(R)$, respectively.

Definition 2.5. Consider the complex $X$ with $X_{n} \in \mathcal{P}(R)$ such that

$$
\mathrm{H}_{n}(X)=\mathrm{H}_{n}\left(\operatorname{Hom}_{R}(X, \mathcal{P}(R))\right)=0
$$

for all $n \in \mathbb{Z}$. The syzygies of this complex are called Gorenstein projective $R$-modules. We denote the class of Gorenstein projective $R$-modules by $\mathcal{G P}$.

An $R$-module $N$ is called Gorenstein injective, if there exists an exact complex $Y$ of injective $R$-modules such that $N$ is isomorphic to a cokernel of $Y$, and

$$
\mathrm{H}_{n}\left(\operatorname{Hom}_{R}(\mathcal{I}(R), Y)\right)=0 .
$$

We denote the class of Gorenstein injective $R$-modules by $\mathcal{G} \mathcal{I}$.

Definition 2.6 ([6]). An $R$-module $M$ is called Ding projective if there exists a $\operatorname{Hom}_{R}(-, \mathcal{F}(R))$-exact exact sequence of projective $R$-modules

$$
\cdots \rightarrow P_{1} \rightarrow P_{0} \rightarrow P^{0} \rightarrow P^{1} \rightarrow \cdots
$$

with $M=\operatorname{Ker}\left(P^{0} \rightarrow P^{1}\right)$. Denote the class of Ding projective $R$-modules by $\mathcal{D P}$.

An $R$-module $N$ is called Ding injective if there exists a $\operatorname{Hom}_{R}(\mathcal{F} \mathcal{I}(R),-)$ exact exact sequence of injective $R$-modules

$$
\cdots \rightarrow I_{1} \rightarrow I_{0} \rightarrow I^{0} \rightarrow I^{1} \rightarrow \cdots
$$

with $N=\operatorname{Coker}\left(I_{1} \rightarrow I_{0}\right)$. Denote the class of Ding injective $R$-modules by $\mathcal{D I}$.

Remark 2.7. Note that every Ding projective (respectively, Ding injective) module is Gorenstein projective (respectively, Gorenstein injective). If $R$ is Noetherian, then any FP-injective module is injective by [12, Thm. 1.6], and so any Gorenstein injective module is Ding injective. Clearly, any Gorenstein projective module over a perfect ring is Ding projective. Also, it follows from [6, Cor. 4.6] that any Gorenstein projective (respectively, Gorenstein injective) module over a Gorenstein ring is Ding projective (respectively, Ding injective).

Definition 2.8. Let $\mathcal{X}$ be a class of $R$-modules and $M$ an $R$-module. An $\mathcal{X}$-resolution of $M$ is a complex of $R$-modules in $\mathcal{X}$ of the form

$$
X=\cdots \rightarrow X_{n} \rightarrow X_{n-1} \rightarrow \cdots \rightarrow X_{1} \rightarrow X_{0} \rightarrow 0
$$

such that $\mathrm{H}_{0}(X) \cong M$ and $\mathrm{H}_{n}(X)=0$ for all $n \geq 1$, and the following exact sequence is the augmented $\mathcal{X}$-resolution of $M$ associated to $X$ :

$$
X^{+}=\cdots \rightarrow X_{n} \rightarrow X_{n-1} \rightarrow \cdots \rightarrow X_{1} \rightarrow X_{0} \rightarrow M \rightarrow 0 .
$$

The $\mathcal{X}$-projective dimension of $M$ is the quantity

$$
\mathcal{X}-\operatorname{pd}_{R}(M)=\inf \left\{\sup \left\{n \geq 0 \mid X_{n} \neq 0\right\} \mid X \text { is an } \mathcal{X} \text {-resolution of } M\right\} .
$$


In particular, one has $\mathcal{X}$-pd $\operatorname{pd}_{R}(0)=-\infty$. The modules of $\mathcal{X}$-projective dimension 0 are the nonzero modules of $\mathcal{X}$. We set

$$
\widetilde{\mathcal{X}}=\text { the subcategory of } R \text {-modules with } \mathcal{X} \text {-pd } \operatorname{pd}_{R}(M)<\infty \text {. }
$$

An $\mathcal{X}$-resolution $X$ of $M$ is proper if the augmented resolution $X^{+}$is $\operatorname{Hom}_{R}(\mathcal{X},-)$-exact. And we set

$\overline{\mathcal{X}}=$ the subcategory of $R$-modules admitting a proper $\mathcal{X}$-resolution.

We define (proper) $\mathcal{X}$-coresolution and $\mathcal{X}$-injective dimension dually. And the $\mathcal{X}$-injective dimension of $M$ is denoted $\mathcal{X}$-id $R(M)$.

Notation 2.9. When $\mathcal{X}$ is the class of projective (Ding projective) $R$-modules, we write $\operatorname{pd}_{R} M\left(\operatorname{Dpd}_{R} M\right)$ for the associated homological dimension and call it the projective (Ding projective) dimension of $M$, respectively. Similarly, the injective (Ding injective) and flat dimensions of $M$ are denoted $\operatorname{id}_{R} M\left(\operatorname{Did}_{R} M\right)$ and $\operatorname{fd}_{R} M$, respectively.

In particular, we abbreviate as follows:

$$
\begin{aligned}
\widetilde{\mathcal{D P}} & =\text { the subcategory of } R \text {-modules with } \operatorname{Dpd}_{R} M<\infty . \\
\widetilde{\mathcal{D I}} & =\text { the subcategory of } R \text {-modules with } \operatorname{Did}_{R} M<\infty .
\end{aligned}
$$

$\overline{\mathcal{D P}}=$ the subcategory of $R$-modules admitting a proper $\mathcal{D} \mathcal{P}$-resolution.

$\overline{\mathcal{D I}}=$ the subcategory of $R$-modules admitting a proper $\mathcal{D} \mathcal{I}$-coresolution.

Definition 2.10. Let $\mathcal{X}$ be any class of $R$-modules and $M$ an $R$-module. An $\mathcal{X}$-precover of $M$ is an $R$-homomorphism $\varphi: X \rightarrow M$, where $X \in \mathcal{X}$ and such that the sequence

$$
\operatorname{Hom}_{R}\left(X^{\prime}, X\right) \stackrel{\operatorname{Hom}_{\mathrm{R}}\left(\mathrm{X}^{\prime}, \varphi\right)}{\longrightarrow} \operatorname{Hom}_{R}\left(X^{\prime}, M\right) \longrightarrow 0
$$

is exact for every $X^{\prime} \in \mathcal{X}$. If, moreover, $\varphi f=\varphi$ for $f \in \operatorname{Hom}_{R}(X, X)$ implies $f$ is an automorphism of $X$, then $\varphi$ is called an $\mathcal{X}$-cover of $M$. Also, an $\mathcal{X}$ preenvelope and $\mathcal{X}$-envelope of $M$ are defined "dually".

\section{Relative cohomology}

It is well known over any associative ring $R$ it is standard to derive $\operatorname{Hom}_{R}(-,-)$ using projective resolutions in the first variable, or injective resolutions in the second variable, and doing this, one obtains $\operatorname{Ext}_{R}^{n}(-,-)$ in both cases. In this section, we examine the situation where projective and injective modules are replaced by Ding projective and Ding injective ones, respectively.

At first, we will need the following:

Lemma 3.1. (1) If $M$ is a Ding projective $R$-module, then $\operatorname{Ext}_{\bar{R}}^{\geq 1}(M, W)=0$ for all $R$-modules $W$ of finite flat or finite injective dimension.

(2) If $N$ is a Ding injective $R$-module, then $\operatorname{Ext}_{\bar{R}}^{\geq 1}(U, N)=0$ for all $R$ modules $U$ of finite projective dimension.

(3) Let $R$ be a left coherent ring. If $N$ is a Ding injective $R$-module, then $\operatorname{Ext}_{\bar{R}}^{\geq 1}(U, N)=0$ for all $R$-modules $U$ of finite FP-injective dimension. 
Proof. (1) For an $R$-module $W$ of finite flat dimension, $\operatorname{Ext}_{R}^{\geq 1}(M, W)=0$ is an immediate consequence of [3, Lem. 2.4(1)].

Assume that $\operatorname{id}_{R} W=m<\infty$. Since $M$ is Ding projective, we have an exact sequence

$$
0 \rightarrow M \rightarrow P^{0} \rightarrow P^{1} \rightarrow \cdots \rightarrow P^{1-m} \rightarrow C \rightarrow 0,
$$

where all $P^{i}$ are projective $R$-modules. Breaking this sequence into short exact ones, we see that $\operatorname{Ext}_{R}^{i}(M, W) \cong \operatorname{Ext}_{R}^{i+m}(C, W)$ for $i>0$, so the Exts vanish as desired since $\operatorname{Ext}_{R}^{i+m}(C, W)=0$ for $i>0$.

(2) The proof is dual to (1).

(3) Note that [11, Thm. 2.4], the proof is similar to that of (1).

Furthermore, we will need the following from [16, Lem. 2.5]:

Proposition 3.2. (1) If $M$ is an $R$-module with $\operatorname{Dpd}_{R} M<\infty$, then there exists a short exact sequence $0 \rightarrow K \rightarrow D \rightarrow M \rightarrow 0$, where $D \rightarrow M$ is a $\mathcal{D P}$-precover of $M$, and $\operatorname{pd}_{R} K=\operatorname{Dpd}_{R} M-1$ (in the case where $M$ is Ding projective, this should be interpreted as $K=0)$.

Consequently, every $R$-module with finite Ding projective dimension has a proper left $\mathcal{D P}$-resolution (that is, there is an inclusion $\widetilde{\mathcal{D P}} \subseteq \overline{\mathcal{D P}}$ ).

(2) If $N$ is an $R$-module with $\operatorname{Did}_{R} N<\infty$, then there exists a short exact sequence $0 \rightarrow N \rightarrow E \rightarrow C \rightarrow 0$, where $N \rightarrow E$ is a $\mathcal{D} \mathcal{I}$-preenvelope of $N$, and $\operatorname{id}_{R} C=\operatorname{Did}_{R} N-1$ (in the case where $N$ is Ding injective, this should be interpreted as $C=0$ ).

Consequently, every $R$-module with finite Ding injective dimension has a proper right $\mathcal{D I}$-coresolution (that is, there is an inclusion $\widetilde{\mathcal{D I}} \subseteq \overline{\mathcal{D I}}$ ).

Lemma 3.3. (1) Let $M \in \widetilde{\mathcal{D P}}$. Then any proper left $\mathcal{D} \mathcal{P}$-resolution $\mathbf{D} \rightarrow M$ of $M$ is $\operatorname{Hom}_{R}(-, \mathcal{D} \mathcal{I})$-exact.

(2) Let $N \in \widetilde{\mathcal{D I}}$. Then any proper right $\mathcal{D I}$-coresolution $N \rightarrow \mathbf{E}$ of $N$ is $\operatorname{Hom}_{R}(\mathcal{D} \mathcal{P},-)$-exact.

Proof. (1) Let $\mathbf{D}=\cdots \rightarrow D_{1} \rightarrow D_{0} \rightarrow M \rightarrow 0$ be a proper left $\mathcal{D} \mathcal{P}$-resolution of $M$. We split the resolution $\mathbf{D}$ into short exact sequences. Hence it suffices to show exactness of $\operatorname{Hom}_{R}(\mathbf{T}, H)$ for all Ding injective $R$-modules $H$ and all short exact sequences

$$
\mathbf{T}=0 \rightarrow L \rightarrow D \rightarrow M \rightarrow 0,
$$

where $D \rightarrow M$ is a $\mathcal{D} \mathcal{P}$-precover of $M$. By Proposition 3.2(1), there is a short exact sequence

$$
\mathbf{T}^{\prime}=0 \longrightarrow L^{\prime} \stackrel{\iota}{\longrightarrow} D^{\prime} \stackrel{\pi}{\longrightarrow} M \longrightarrow 0,
$$

where $\pi: D^{\prime} \rightarrow M$ is a $\mathcal{D} \mathcal{P}$-precover of $M$ and $\operatorname{pd}_{R} L^{\prime}<\infty$.

By [8, Prop. 2.2], the complexes $\mathbf{T}$ and $\mathbf{T}^{\prime}$ are homotopically equivalent, and thus so are the complexes $\operatorname{Hom}_{R}(\mathbf{T}, H)$ and $\operatorname{Hom}_{R}\left(\mathbf{T}^{\prime}, H\right)$ for every (Ding injective) $R$-module $H$. Hence it suffices to show the exactness of $\operatorname{Hom}_{R}\left(\mathbf{T}^{\prime}, H\right)$ whenever $H$ is Ding injective. 
Now let $H$ be any Ding injective $R$-module. We need to prove the exactness of

$$
\operatorname{Hom}_{R}\left(D^{\prime}, H\right) \stackrel{\operatorname{Hom}_{\mathrm{R}}(\iota, \mathrm{H})}{\longrightarrow} \operatorname{Hom}_{R}\left(L^{\prime}, H\right) \longrightarrow 0 .
$$

To show this, let $f: L^{\prime} \rightarrow H$ be any homomorphism. We wish to find $g: D^{\prime} \rightarrow$ $H$ such that $g \iota=f$. Now pick an exact sequence

$$
0 \longrightarrow H^{\prime} \longrightarrow I \stackrel{\alpha}{\longrightarrow} H \longrightarrow 0,
$$

where $I$ is injective, and $H^{\prime}$ is Ding injective. Since $H^{\prime}$ is Ding injective and $\operatorname{pd}_{R} L^{\prime}<\infty$, we get $\operatorname{Ext}_{R}^{1}\left(L^{\prime}, H^{\prime}\right)=0$ by Lemma $3.1(2)$, and thus a lifting $\mu: L^{\prime} \rightarrow I$ with $\alpha \mu=f:$

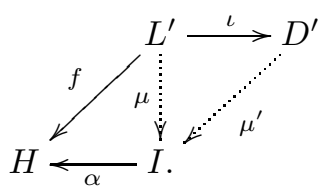

Next, injectivity of $I$ gives $\mu^{\prime}: D^{\prime} \rightarrow I$ with $\mu^{\prime} \iota=\mu$. Now $g=\alpha \mu^{\prime}: D^{\prime} \rightarrow H$ is the desired map.

(2) Dual the proof of (1).

Definition 3.4. Let $M \in \overline{\mathcal{D P}}$ and consider a proper left $\mathcal{D} \mathcal{P}$-resolution $\mathbf{D} \rightarrow$ $M$. For every $n \in \mathbb{Z}$ and every $R$-module $N$, define a relative cohomology group

$$
\operatorname{Ext}_{\mathcal{D} \mathcal{P}}^{n}(M, N)=\mathrm{H}^{n}\left(\operatorname{Hom}_{R}(\mathbf{D}, N)\right) .
$$

Similarly, choosing for each $N \in \overline{\mathcal{D I}}$ a proper right $\mathcal{D} \mathcal{I}$-coresolution $N \rightarrow \mathbf{E}$, we define for each $n \in \mathbb{Z}$ and each $R$-module $M$ a relative cohomology group

$$
\operatorname{Ext}_{\mathcal{D} \mathcal{I}}^{n}(M, N)=\mathrm{H}^{n}\left(\operatorname{Hom}_{R}(M, \mathbf{E})\right) \text {. }
$$

Recall that a ring $R$ is called an $n$-FC ring if it is both left and right coherent and FP-id $\left({ }_{R} R\right)$ and FP-id $\left(R_{R}\right)$ are both less than or equal to $n$. A ring $R$ is called Ding-Chen if it is an $n$-FC ring for some non-negative integer $n$. It follows from [13, Cor. 4.5] and [17, Thm. 3.6] that over a Ding-Chen ring $R$, for any $R$-modules $M, N$ and any $i \in \mathbb{Z}$ we have

$$
\operatorname{Ext}_{\mathcal{D} \mathcal{P}}^{i}(M, N) \cong \operatorname{Ext}_{\mathcal{D} \mathcal{I}}^{i}(M, N) \text {. }
$$

Though we don't know whether or not every $R$-module has finite Ding injective (or projective) dimension when $R$ is a Ding-Chen ring (see [3, Thm. 3.6] and [11, Lem. 3.1] for partial answer), the above lemma can be stated in the language of Enochs and Jenda [5, Thm. 8.2.13] as follows: the bifunctor $\operatorname{Hom}_{R}(-,-)$ is right balanced by $\widetilde{\mathcal{D P}} \times \widetilde{\mathcal{D I}}$. So we have the following interesting result.

Theorem 3.5. Let $M \in \widetilde{\mathcal{D P}}$ and $N \in \widetilde{\mathcal{D I}}$. Then for all $i \in \mathbb{Z}$, we have isomorphisms

$$
\operatorname{Ext}_{\mathcal{D} \mathcal{P}}^{i}(M, N) \cong \operatorname{Ext}_{\mathcal{D} \mathcal{I}}^{i}(M, N) .
$$

Proof. Use [8, Thm. 2.6]. 
Definition 3.6 (Definition of Dext). Let $M \in \widetilde{\mathcal{D P}}$ and $N \in \widetilde{\mathcal{D I}}$. Then we write

$$
\operatorname{Dext}_{R}^{n}(M, N):=\operatorname{Ext}_{\mathcal{D} \mathcal{P}}^{n}(M, N) \cong \operatorname{Ext}_{\mathcal{D} \mathcal{I}}^{n}(M, N)
$$

for the isomorphic abelian groups in Theorem 3.5 above.

The following proposition corresponds to [2, (2.b), (4.2), (4.4), (4.6)], whose proofs extend verbatim to the more general setting below.

Proposition 3.7. (1) $\operatorname{Dext}_{R}^{0}(-,-)=\operatorname{Hom}_{R}(-,-)$.

(2) If the exact sequence $0 \rightarrow M^{\prime} \rightarrow M \rightarrow M^{\prime \prime} \rightarrow 0$ of $R$-modules in $\overline{\mathcal{D P}}$ is $\operatorname{Hom}_{R}(\mathcal{D} \mathcal{P},-)$-exact, then for each $R$-module $N$ there is a long exact sequence

$$
\cdots \rightarrow \operatorname{Ext}_{\mathcal{D} \mathcal{P}}^{i}(M, N) \rightarrow \operatorname{Ext}_{\mathcal{D P}}^{i}\left(M^{\prime}, N\right) \rightarrow \operatorname{Ext}_{\mathcal{D} \mathcal{P}}^{i+1}\left(M^{\prime \prime}, N\right) \rightarrow \cdots .
$$

(3) If the exact sequence $0 \rightarrow N^{\prime} \rightarrow N \rightarrow N^{\prime \prime} \rightarrow 0$ of $R$-modules is $\operatorname{Hom}_{R}(\mathcal{D P},-)$-exact, then for each $R$-module $M \in \overline{\mathcal{D P}}$ there is a long exact sequence

$$
\cdots \rightarrow \operatorname{Ext}_{\mathcal{D P}}^{i}(M, N) \rightarrow \operatorname{Ext}_{\mathcal{D} \mathcal{P}}^{i}\left(M, N^{\prime \prime}\right) \rightarrow \operatorname{Ext}_{\mathcal{D P}}^{i+1}\left(M, N^{\prime}\right) \rightarrow \cdots .
$$

(4) There are natural transformations

$$
\operatorname{Dext}_{R}^{i}(-,-) \longrightarrow \operatorname{Ext}_{R}^{i}(-,-)
$$

which are also natural in the long exact sequences as in (2) and (3) above.

In the following, we want to compare Dext with the classical Ext.

Theorem 3.8. Let $M, N$ be any R-modules. Then the following conclusions hold:

(1) There are natural isomorphisms $\operatorname{Ext}_{\mathcal{D} \mathcal{P}}^{n}(M, N) \cong \operatorname{Ext}_{R}^{n}(M, N)$ under each of the conditions
(a) $\operatorname{pd}_{R} M<\infty \quad$ or
(b) $M \in \overline{\mathcal{D P}}$ and $\operatorname{id}_{R} N<\infty$.

(2) There are natural isomorphisms $\operatorname{Ext}_{\mathcal{D} \mathcal{I}}^{n}(M, N) \cong \operatorname{Ext}_{R}^{n}(M, N)$ under each of the conditions

$$
\text { (a) } \operatorname{id}_{R} N<\infty \quad \text { or } \quad \text { (b) } N \in \overline{\mathcal{D I}} \text { and } \operatorname{pd}_{R} M<\infty \text {. }
$$

(3) Assume that $M \in \widetilde{\mathcal{D P}}$ and $N \in \widetilde{\mathcal{D I}}$. If either $\operatorname{pd}_{R} M<\infty$ or $\operatorname{id}_{R} N<\infty$, then

$$
\operatorname{Dext}_{R}^{n}(M, N) \cong \operatorname{Ext}_{R}^{n}(M, N)
$$

is functorial in $M$ and $N$.

Proof. (1) (a) Assume that $\operatorname{pd}_{R} M<\infty$, and pick any projective resolution $\mathbf{P}$ of $M$. Since $\mathbf{P}$ is also a proper left $\mathcal{D} \mathcal{P}$-resolution of $M$, and thus

$$
\operatorname{Ext}_{\mathcal{D P}}^{n}(M, N)=\mathrm{H}^{n}\left(\operatorname{Hom}_{R}(\mathbf{P}, N)\right)=\operatorname{Ext}_{R}^{n}(M, N) .
$$

(b) Assume that $M \in \overline{\mathcal{D P}}$ and $\operatorname{id}_{R} N=m<\infty$. By Lemma 3.1(1), we see that $\operatorname{Ext}_{R}^{i}(D, N)=0$ for every Ding projective $R$-module $D$ and all $i>0$. 
Therefore [7, Chapter III, Prop. $1.2 \mathrm{~A}]$ implies that $\operatorname{Ext}_{R}^{i}(-, N)$ can be computed using (proper) left $\mathcal{D} \mathcal{P}$-resolutions of the argument in the first variable, as desired.

The proof of (2) is similar.

The claim (3) is a direct consequence of (1) and (2), together with the Definition 3.6 of $\operatorname{Dext}_{R}^{i}(-,-)$.

In [8], Holm proved that one can compute right derived functors of $\operatorname{Hom}_{R}(M, N)$ using either a proper left $\mathcal{G P}$-resolution of $M$ or a proper right $\mathcal{G I}$-coresolution of $N$, these derived functors be denoted by $\operatorname{GExt}_{R}^{i}(M, N)$. Using the theorem above together with [8, Thm. 3.8], we have the following

Corollary 3.9. Let $M \in \widetilde{\mathcal{D P}}$ and $N \in \widetilde{\mathcal{D I}}$. If $\operatorname{pd}_{R} M<\infty$ or $\operatorname{id}_{R} N<\infty$, then there are natural isomorphisms

$$
\operatorname{Dext}_{R}^{i}(M, N) \cong \operatorname{GExt}_{R}^{i}(M, N) \text {. }
$$

\section{Tate cohomology}

In this section, we define and study a Tate cohomology theory for complexes of Ding homological modules over associative rings.

The following notions are fundamental to our investigation.

Definition 4.1. Let $I$ be an $R$-complex. Following [1], $I$ is called DG-injective if $\operatorname{Hom}_{R}(-, I)$ preserves injective quasi-isomorphisms.

Definition 4.2. A DG-injective resolution of a complex $X$ is a quasi-isomorphism of complexes $\lambda: X \rightarrow I$, with $I$ DG-injective.

Remark 4.3. By [1, (1.6)], every complex $X$ has a injective DG-injective resolution $X \rightarrow I$.

Definition 4.4. A complex of $R$-modules $S$ is said to be totally $\mathcal{F} \mathcal{I}$-acyclic if the following conditions are satisfied.

(1) $S_{n}$ is injective for every $n \in \mathbb{Z}$.

(2) $S$ is exact.

(3) $\operatorname{Hom}_{R}(E, S)$ is exact for every FP-injective $R$-module $E$.

Remark 4.5. (1) By the definition of Ding injective $R$-module $M$, there exists a totally $\mathcal{F} \mathcal{I}$-acyclic complex $S$ with $C_{0}(S)=M$.

(2) Recall that an $R$-complex $S$ is called totally acyclic if $S_{n} \in \mathcal{I}$ and $\mathrm{H}_{n}(S)=0=\mathrm{H}_{n}\left(\operatorname{Hom}_{R}(E, S)\right)$ for each $E \in \mathcal{I}$ and each $n \in \mathbb{Z}$ [14]. Since $\mathcal{I} \subseteq \mathcal{F} \mathcal{I}$, then every totally $\mathcal{F} \mathcal{I}$-acyclic complex is totally acyclic.

Definition 4.6. Let $X$ be an $R$-complex. A Tate $\mathcal{F} \mathcal{I}$-coresolution of $X$ is a diagram $X \stackrel{\lambda}{\longrightarrow} I \stackrel{\nu}{\longrightarrow} S$ of morphisms of complexes, where $S$ is a totally $\mathcal{F} \mathcal{I}$ acyclic complex, $\lambda$ is a $D G$-injective resolution of $X$, and $\nu_{i}$ is bijective for all $i \ll 0$. 
The next result is the key for well-definedness and functoriality of Tate cohomology.

Proposition 4.7. Let $X \stackrel{\lambda}{\longrightarrow} I \stackrel{\nu}{\longrightarrow} S$ and $X^{\prime} \stackrel{\lambda^{\prime}}{\longrightarrow} I^{\prime} \stackrel{\nu^{\prime}}{\longrightarrow} S^{\prime}$ be Tate $\mathcal{F} \mathcal{I}$-coresolutions. For each morphism of complexes $\mu: X \rightarrow X^{\prime}$ there is a morphism $\bar{\mu}: I \rightarrow I^{\prime}$, unique up to homotopy, making the left-most square in the next diagram commute up to homotopy,

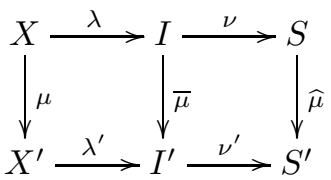

and for each choice of $\bar{\mu}$ there exists a unique up to homotopy morphism $\widehat{\mu}$, making the right-most square commute up to homotopy. Moreover, if $\lambda$ and $\nu$ are injective, then $\bar{\mu}$ and respectively $\widehat{\mu}$ can be chosen such that the left-most square and respectively the right-most square commute.

If $\mu=i d^{X}$, then $\bar{\mu}$ and $\widehat{\mu}$ are homotopy equivalences.

Proof. The proof is almost dual to that of [2, (5.3)].

Definition 4.8. Let $X$ be an $R$-complex. The Ding injective dimension of $X$ is defined by

$$
\mathcal{D} \mathcal{I} \text {-id }{ }_{R} X=\inf \left\{\begin{array}{l|l}
-n \in \mathbb{Z} & \begin{array}{l}
X \stackrel{\lambda}{\longrightarrow} I \stackrel{\nu}{\longrightarrow} S \text { is a Tate } \mathcal{F} \mathcal{I} \text {-coresolution } \\
\text { with } \nu_{i}: I_{i} \rightarrow S_{i} \text { bijective for each } i \leq-n
\end{array}
\end{array}\right\} .
$$

The next assertions follow from the definition.

Remark 4.9. (1) Complex $X$ is exact if and only if $\mathcal{D} \mathcal{I}$-id ${ }_{R} X=-\infty$.

(2) For every $k \in \mathbb{Z}, \mathcal{D} \mathcal{I}$-id ${ }_{R}\left(\Sigma^{k} X\right)=\mathcal{D} \mathcal{I}$-id $R+k$.

(3) Let $M$ be an $R$-module. Then $\mathcal{D} \mathcal{I}$-id ${ }_{R}(\underline{M})=\operatorname{Did}_{R} M$.

By [15, Thm. 5.3.15], complexes admitting Tate $\mathcal{F} \mathcal{I}$-coresolutions are precisely these $R$-complexes of finite Ding injective dimension. This allows us to define our Tate cohomology functors using the coresolutions.

We let $\mathcal{C}(\widetilde{\mathcal{D I}})$ denote the class of complexes of finite Ding injective dimension.

Definition 4.10. Let $N \in \mathcal{C}(\widetilde{\mathcal{D I}})$, and choose a Tate $\mathcal{F} \mathcal{I}$-coresolution

$$
N \stackrel{\lambda}{\longrightarrow} I \stackrel{\nu}{\longrightarrow} S \text {. }
$$

Then for each $R$-complex $M$ and each $n \in \mathbb{Z}$, define a Tate cohomology group by the equality

$$
\overline{\operatorname{Dext}}_{R}^{n}(M, N)=\mathrm{H}^{-n}\left(\operatorname{Hom}_{R}(M, S)\right) .
$$

The morphism

$$
\operatorname{Hom}_{R}(M, \nu): \operatorname{Hom}_{R}(M, I) \longrightarrow \operatorname{Hom}_{R}(M, S)
$$


RELATIVE AND TATE COHOMOLOGY OF DING MODULES AND COMPLEXES 831

induces for every $n \in \mathbb{Z}$ a homomorphism of abelian groups

$$
\overline{\mathrm{D}}_{R}^{n}(M, N): \operatorname{Ext}_{R}^{n}(M, N) \longrightarrow \overline{\operatorname{Dext}}_{R}^{n}(M, N) .
$$

Proposition 4.11. The assignment $(M, N) \mapsto \overline{\operatorname{Dext}}_{R}^{n}(M, N)$ defines a functor

$$
\overline{\operatorname{Dext}}_{R}^{n}(-,-): \mathcal{C} \times \mathcal{C}(\widetilde{\mathcal{D I}}) \longrightarrow \mathcal{M}(\mathbb{Z}),
$$

and the maps $\overline{\mathrm{D}}_{R}^{n}(M, N)$ yield a morphism of functors

such that the following hold:

$$
\overline{\mathrm{D}}_{R}^{n}(-,-): \operatorname{Ext}_{R}^{n}(-,-) \longrightarrow \overline{\operatorname{Dext}}_{R}^{n}(-,-)
$$

(1) The functors $\overline{\operatorname{Dext}}_{R}^{n}(-,-)$ and the morphisms $\overline{\mathrm{D}}_{R}^{n}(-,-)$ are independent of choices of coresolutions and liftings.

(2) If $M$ and $N$ are $R$-modules with $\operatorname{Did}_{R} N=g<\infty$, then the natural map $\overline{\mathrm{D}}_{R}^{n}(M, N)$ is equal to 0 for $n<0$ and is bijective for $n>g$.

(3) For all $n, j \in \mathbb{Z}$, there is an isomorphism

$$
\overline{\operatorname{Dext}}_{R}^{n}\left(-, \Sigma^{-j} N\right) \cong \overline{\operatorname{Dext}}_{R}^{n-j}(-, N) .
$$

(4) For any finite set of complexes $\left\{M^{i}\right\}_{i \in I}$ and any family of complexes $\left\{N^{j}\right\}_{j \in J}$ of finite Ding injective dimensions, there is a natural isomorphism

$$
\overline{\operatorname{Dext}}_{R}^{n}\left(\oplus_{i \in I} M^{i}, \Pi_{j \in J} N^{j}\right) \cong \Pi_{(i, j) \in I \times J} \overline{\operatorname{Dext}}_{R}^{n}\left(M^{i}, N^{j}\right) \text { for each } n \in \mathbb{Z} \text {. }
$$

Proof. The naturality of $\overline{\operatorname{Dext}}_{R}^{n}(-,-)$ and $\overline{\mathrm{D}}_{R}^{n}(-,-)$ follows from Proposition 4.7 by applying the chosen Tate $\mathcal{F} \mathcal{I}$-coresolutions of $N$ and $N^{\prime}$. Their independence from the choice of coresolutions and liftings follows from the last part of the same Proposition. The last assertions follow from the definition of Tate cohomology $\overline{\operatorname{Dext}}_{R}^{n}$.

The Tate cohomology theory is rigid in the sense (that is when the complex is left-bounded) that vanishing of any one of these functors implies the vanishing of all of them and their vanishing characterizes complexes of finite injective dimension.

Proposition 4.12. Let $M \in \mathcal{C}(\widetilde{\mathcal{D I}})$. The following are equivalent.

(i) $\operatorname{id}_{R} M<\infty$.

(ii) $\overline{\operatorname{Dext}}_{R}^{i}(-, M)=0$ for some $i \in \mathbb{Z}$.

(ii) $\overline{\operatorname{Dext}}_{R}^{i}(-, M)=0$ for all $i \in \mathbb{Z}$.

When $\sup M<\infty$, the properties above are also equivalent to the following.

(iii) $\overline{\operatorname{Dext}}_{R}^{i}(M,-)=0$ for some $i \in \mathbb{Z}$.

(iii) $\overline{\operatorname{Dext}}_{R}^{i}(M,-)=0$ for all $i \in \mathbb{Z}$.

(iv) $\overline{\operatorname{Dext}}_{R}^{0}(M, M)=0$.

Proof. (i) $\Rightarrow(\text { ii })^{\prime}$. If $\operatorname{id}_{R} M<\infty$, then $M \stackrel{\lambda}{\rightarrow} I \stackrel{\nu}{\rightarrow} 0$ is a Tate $\mathcal{F} \mathcal{I}$-coresolution for any DG-injective resolution $M \stackrel{\lambda}{\rightarrow} I$, so the groups $\overline{\operatorname{Dext}}_{R}^{i}(-, M)=\mathrm{H}^{-i}$ $\left(\operatorname{Hom}_{R}(-, 0)\right)$ vanish for all $i \in \mathbb{Z}$. 
(ii) ${ }^{\prime} \Rightarrow$ (ii) is clear.

(ii) $\Rightarrow\left(\right.$ i). Choose a Tate $\mathcal{F} \mathcal{I}$-coresolution $M \stackrel{\lambda}{\rightarrow} I \stackrel{\nu}{\rightarrow} S$. Set $K=Z_{-i}(S)$ and let $\alpha: S_{-(i-1)} \rightarrow K$ be the canonical surjection. Since $0=\overline{\operatorname{Dext}}_{R}^{i}(M,-)=$ $\mathrm{H}^{-i}\left(\operatorname{Hom}_{R}(-, S)\right)$, the $\operatorname{map}_{\operatorname{Hom}_{R}}(K, \alpha): \operatorname{Hom}_{R}\left(K, S_{-(i-1)}\right) \rightarrow \operatorname{Hom}_{R}(K, K)$ is surjective. This means that $\alpha$ splits, so $K$ is injective. Induction on $j$ shows that $Z_{j}(S)$ is injective for all $j \leq-i$. By definition, $Z_{j}(I) \cong Z_{j}(S)$ for all $j \leq-\mathcal{D} \mathcal{I}$-id $\operatorname{id}_{R} M$, so $Z_{j}(I)$ is injective for all $j \leq \min \left\{-i,-\mathcal{D} \mathcal{I}\right.$-id id $\left._{R} M\right\}$. From [1, (Thm. $2.4 \mathrm{I})$ ], we get $\operatorname{id}_{R} M<\infty$.

For the rest of the proof we assume sup $M<\infty$.

(i) $\Rightarrow(\text { iii })^{\prime}$. By $[1,($ Thm. $2.4 \mathrm{I})]$, choose a bounded DG-injective resolution $M \rightarrow I$. Now, apply [15, Lem. 5.3.3].

(iii) ${ }^{\prime} \Rightarrow$ (iii) is clear.

(iii) $\Rightarrow$ (iv) follows from the isomorphism $\overline{\operatorname{Dext}}_{R}^{i}\left(M, \Sigma^{-i} M\right) \cong \overline{\operatorname{Dext}}_{R}^{0}(M, M)$.

(iv) $\Rightarrow$ (i). By $[1$, (Thm. 2.4 I)], choose a bounded above DG-injective resolution $M \rightarrow I$ and by $[15$, Thm. 5.3.15] choose a Tate $\mathcal{F} \mathcal{I}$-coresolution $M \stackrel{\lambda}{\rightarrow} I \stackrel{\nu}{\rightarrow} S$ with $\nu_{j}=i d^{S_{j}}$ for all $j \ll 0$. Then we have $\overline{\operatorname{Dext}}_{R}^{0}(M, M) \cong$ $\mathrm{H}^{0}\left(\operatorname{Hom}_{R}(I, S)\right)=0$ since $I$ is a DG-injective $R$-complex. As $\nu$ is in $\mathrm{B}_{0}\left(\operatorname{Hom}_{R}(I, S)\right)$, there exists a $\sigma \in \operatorname{Hom}_{R}(I, S)_{0}$ such that $\partial(\sigma)=\nu$ in $\operatorname{Hom}_{R}(I, S)$. Since $\nu_{j}=i d^{S_{j}}$ for all $j \ll 0$ and the complex $S$ is exact, we obtain the map $\operatorname{Ker}\left(\partial_{-(j+1)}^{S}\right) \rightarrow S_{-(j+1)}$ splits for all $j \ll 0$, so $\operatorname{id}_{R} M<\infty$ by [1, (Thm. 2.4 I)].

Remark 4.13. Dually, for any $R$-complex $M$, one can define Tate $\mathcal{F}$-resolution $T \stackrel{u}{\rightarrow} P \stackrel{\pi}{\rightarrow} M$ and Ding projective dimension $\mathcal{D} \mathcal{P}-\operatorname{pd}_{R} M$. Dual the above results, we have that the complex of finite Ding projective dimension admits a Tate $\mathcal{F}$-resolution. Accordingly, for any $R$-complex $N$ and any $n \in \mathbb{Z}$, the $n$th Tate cohomology group $\widehat{\operatorname{Dext}}_{R}^{n}(M, N)$ is defined as

$$
\widehat{\operatorname{Dext}}_{R}^{n}(M, N)=\mathrm{H}^{n}\left(\operatorname{Hom}_{R}(T, N)\right),
$$

which is independent of choices of resolutions and liftings by dualing Proposition 4.11 .

\section{Avramov-Martsinkovsky type exact sequence}

In this section $M$ is a left $R$-module.

Recall that for any $R$-module $M$ with $\operatorname{Gpd}_{R} M<\infty$, there exists a complete resolution, that is, a diagram $T \stackrel{\vartheta}{\rightarrow} P \stackrel{\pi}{\rightarrow} M$ where $P \stackrel{\pi}{\rightarrow} M$ is a projective resolution, $T$ is a totally acyclic complex (see Remark 4.5(2)), and $\vartheta_{i}$ is bijective for all $i \gg 0$. For any $R$-module $N$ and each $n \in \mathbb{Z}$, the $n$th Tate cohomology group is defined as

$$
\widehat{\operatorname{Ext}}_{R}^{n}(M, N)=\mathrm{H}^{n}\left(\operatorname{Hom}_{R}(T, N)\right),
$$


which is independent of choices of resolutions and liftings. The close relations between absolute, relative and Tate cohomology are illuminated by an Avramov-Martsinkovsky type exact sequence (see [2, Thm. 7.1]) as follows:

$$
\begin{aligned}
0 \longrightarrow \operatorname{Ext}_{\mathcal{G P}}^{1}(M, N) \longrightarrow \operatorname{Ext}_{R}^{1}(M, N) \longrightarrow \widehat{\operatorname{Ext}}_{R}^{1}(M, N) \longrightarrow \operatorname{Ext}_{\mathcal{G P}}^{2}(M, N) \longrightarrow \cdots \\
\longrightarrow \operatorname{Ext}_{\mathcal{G P}}^{i}(M, N) \longrightarrow \operatorname{Ext}_{R}^{i}(M, N) \longrightarrow \widehat{\operatorname{Ext}}_{R}^{i}(M, N) \longrightarrow \operatorname{Ext}_{\mathcal{G P}}^{i+1}(M, N) \longrightarrow \cdots
\end{aligned}
$$

In [9] Iacob gave another way to prove the existence of above exact sequence. Recently, Ren, Liu and Yang in [13] considered Iacob's generalized Tate cohomology for Ding modules as follows:

Definition 5.1. Let $M$ be an $R$-module admitting a proper left $\mathcal{D} \mathcal{P}$-resolution and $N$ be an arbitrary $R$-module. Let $P^{\bullet} \rightarrow M \rightarrow 0$ and $D^{\bullet} \rightarrow M \rightarrow 0$ be a projective and a proper left $\mathcal{D} \mathcal{P}$-resolution of $M$ respectively. Then there exists a morphism $f: P^{\bullet} \rightarrow D^{\bullet}$. Define a Tate cohomology with respect to Ding projective modules as

$$
\widehat{\operatorname{Ext}}_{\mathcal{D P}}^{n}(M, N)=\mathrm{H}^{n+1}\left(\operatorname{Hom}_{R}(\operatorname{Cone}(f), N)\right) .
$$

Since every $R$-module with finite Ding projective dimension has a proper left $\mathcal{D} \mathcal{P}$-resolution(see Proposition 3.2(1)), the following result shows that the Tate cohomology of ours and the generalized Tate cohomology of Ren et al.'s are identical under the following general condition.

Theorem 5.2. Let $M \in \widetilde{\mathcal{D P}}$. Then for each $R$-module $N$ and each $n \geq 1$, we have

$$
\widehat{\operatorname{Dext}}_{R}^{n}(M, N) \cong \widehat{\operatorname{Ext}}_{\mathcal{D P}}^{n}(M, N) \text {. }
$$

Proof. Set $\operatorname{Dpd}_{R} M=g<\infty$. We want to construct a Tate $\mathcal{F}$-resolution of $M$.

$$
\text { If } 0 \rightarrow C \stackrel{i}{\rightarrow} P_{g-1} \stackrel{f_{g-1}}{\longrightarrow} P_{g-2} \stackrel{f_{g-2}}{\longrightarrow} \cdots \rightarrow P_{1} \stackrel{f_{1}}{\rightarrow} P_{0} \stackrel{\pi}{\rightarrow} M \rightarrow 0 \text { is a partial }
$$
projective resolution of $M$ then $C$ is a Ding projective $R$-module by [10, Thm. $2.4]$. Hence there exists a totally $\mathcal{F}$-acyclic complex

$$
\mathbf{T}=\cdots \longrightarrow P^{-2} \stackrel{d_{-2}}{\longrightarrow} P^{-1} \stackrel{d_{-1}}{\longrightarrow} P^{0} \stackrel{d_{0}}{\longrightarrow} P^{1} \longrightarrow \cdots
$$

such that $C=\operatorname{Ker} d_{0}$.

Since $C=\operatorname{Im} d_{1}=\operatorname{Ker} f_{g-1}$, the complex

$\mathbf{P}=\cdots \rightarrow P^{-2} \stackrel{d_{-2}}{\longrightarrow} P^{-1} \stackrel{i \circ d_{-1}}{\longrightarrow} P_{g-1} \stackrel{f_{g-1}}{\longrightarrow} P_{g-2} \stackrel{f_{g-2}}{\longrightarrow} \cdots \rightarrow P_{1} \stackrel{f_{1}}{\longrightarrow} P_{0} \stackrel{\pi}{\longrightarrow} M \longrightarrow 0$

is a projective resolution of $M$.

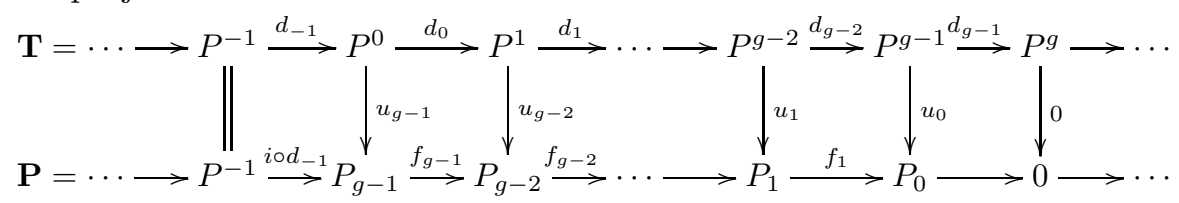

Since $P^{g-1}$ is flat, the complex $\operatorname{Hom}_{R}\left(\mathbf{T}, P^{g-1}\right)$ is exact, there exists $u_{g-1} \in$ $\operatorname{Hom}_{R}\left(P^{0}, P^{g-1}\right)$ such that $i \circ d_{-1}=u_{g-1} \circ d_{-1}$. Similarly there exist $u_{g-2}, \ldots$, 
$u_{1}, u_{0}$ that make the diagram commutative. Since $u: \mathbf{T} \rightarrow \mathbf{P}$ is a morphism of complexes (with $u_{0}, \ldots, u_{g-1}$ as above and $u_{n}=i d_{P^{g-1-n}}$ for $n \geq g$ ) with $u_{n}$ is bijective for $n \geq g$, it follows that $\mathbf{T} \stackrel{u}{\longrightarrow} \mathbf{P} \stackrel{\pi}{\longrightarrow} M$ is a Tate $\mathcal{F}$-resolution.

Now, we use the projective resolution $\mathbf{P}$ and the Tate $\mathcal{F}$-resolution $\mathbf{T}$ to construct a proper left $\mathcal{D} \mathcal{P}$-resolution of $M$.

Let $D=\operatorname{Im} d_{g-1}$. Then $D$ is a Ding projective $R$-module by $[3, \operatorname{Rem} .2 .2]$ (3) and there is a commutative diagram:

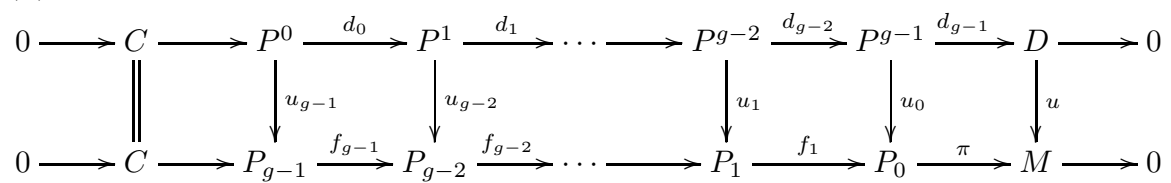

with $u$ defined by $u\left(d_{g-1}(x)\right)=\pi\left(u_{0}(x)\right)$.

Since both rows are exact complexes, the associated mapping cone

$$
\begin{aligned}
\mathbf{C}=0 \longrightarrow & \longrightarrow \longrightarrow P_{1} \oplus P^{g-1} \stackrel{\delta_{g-1}}{\longrightarrow} P_{0} \oplus D \stackrel{\beta}{\longrightarrow} P^{0} \stackrel{\delta_{0}}{\longrightarrow} P_{g-1} \oplus P^{1} \stackrel{\delta_{1}}{\longrightarrow} P_{g-2} \oplus P^{2} \longrightarrow \cdots \\
& \longrightarrow
\end{aligned}
$$

is also an exact complex, so we have an exact complex

$$
\mathbf{D}=0 \rightarrow P^{0} \stackrel{\overline{\delta_{0}}}{\rightarrow} P_{g-1} \oplus P^{1} \stackrel{\delta_{1}}{\rightarrow} P_{g-2} \oplus P^{2} \rightarrow \cdots \rightarrow P_{0} \oplus D \stackrel{\beta}{\rightarrow} M \rightarrow 0 .
$$

Let $L$ be a Ding projective $R$-module. Since $\operatorname{fd}_{R} \operatorname{Ker} \beta<\infty$, we have $\operatorname{Ext}_{R}^{1}(L$, $\operatorname{Ker} \beta)=0$ by Lemma 3.1(1). The sequence $0 \rightarrow \operatorname{Ker} \beta \rightarrow P_{0} \oplus D \rightarrow M \rightarrow 0$ is exact, so we have the associated exact sequence $0 \rightarrow \operatorname{Hom}_{R}(L, \operatorname{Ker} \beta) \rightarrow$ $\operatorname{Hom}_{R}\left(L, P_{0} \oplus D\right) \rightarrow \operatorname{Hom}_{R}(L, M) \rightarrow \operatorname{Ext}_{R}^{1}(L, \operatorname{Ker} \beta)=0$. Thus $P_{0} \oplus D \rightarrow M$ is a Ding projective precover. Similarly $P_{1} \oplus P^{g-1} \rightarrow \operatorname{Ker} \beta, \cdots, P^{0} \rightarrow \operatorname{Ker} \delta_{1}$ is a Ding projective precover respectively. So $\mathbf{D}$ is a proper left $\mathcal{D} \mathcal{P}$-resolution of $M$.

There is a map of complexes $e: \mathbf{P} \rightarrow \mathbf{D}$ as follows:

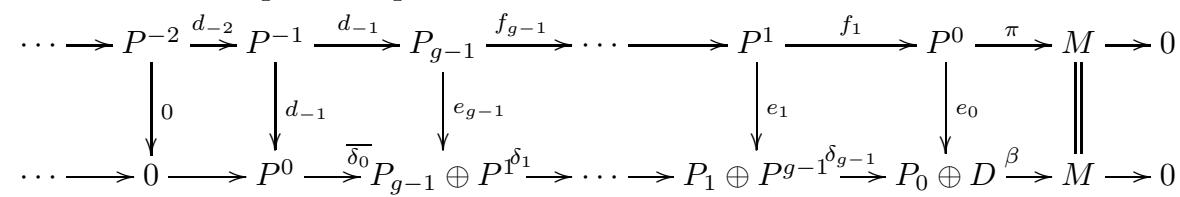

with

$$
\left\{\begin{array}{cl}
e_{0}: P_{0} \rightarrow P_{0} \oplus D, & e_{0}(x)=(x, 0), \\
e_{j}: P_{j} \rightarrow P_{j} \oplus P^{g-j}, & e_{j}(x)=(x, 0), \quad 1 \leq j \leq g-1 .
\end{array}\right.
$$

By assembling the information above, we have $\mathbf{P}$ is a projective resolution, $\mathbf{D}$ is a proper left $\mathcal{D} \mathcal{P}$-resolution of $M$ and $e: \mathbf{P} \rightarrow \mathbf{D}$ is a chain map induced by $i d_{M}$, so $\widehat{\operatorname{Ext}}_{\mathcal{D P}}^{n}(M, N)=\mathrm{H}^{n+1}\left(\operatorname{Hom}_{R}(\operatorname{Cone}(e), N)\right)$ for all $n \geq 0$ by Definition 5.1 .

Let

$$
\overline{\mathbf{T}}=\cdots \longrightarrow P^{-1} \stackrel{d_{-1}}{\longrightarrow} P^{0} \stackrel{d_{0}}{\longrightarrow} \cdots \longrightarrow P^{g-2} \stackrel{d_{g-2}}{\longrightarrow} P^{g-1} \stackrel{d_{g-1}}{\longrightarrow} D \longrightarrow 0 .
$$

We prove that Cone $(e)$ and $\bar{T}[1]$ are homotopically equivalent. 
RELATIVE AND TATE COHOMOLOGY OF DING MODULES AND COMPLEXES 835

There is a map of complexes $\alpha: \bar{T}[1] \rightarrow$ Cone $(e)$ with $\alpha_{0}: P^{0} \rightarrow P^{0} \oplus P_{g-1}, \alpha_{0}(x)=\left(x,-u_{g-1}(x)\right), x \in P^{0}$,

$\alpha_{j}: P^{j} \rightarrow P_{g-j} \oplus P^{j} \oplus P_{g-j-1}, \alpha_{j}(x)=\left(0, x,-u_{g-j-1}(x)\right), x \in P^{j}, 1 \leq$ $j \leq g-1$,

$\alpha^{\prime}: D \rightarrow P_{0} \oplus D, \alpha^{\prime}(x)=(0, x), x \in D$

$$
\left\{\begin{array}{cl}
\alpha_{j}=-i d_{P^{j}}, & \text { if } j \leq-1 \text { is odd } \\
\alpha_{j}=i d_{P^{j}}, & \text { if } j \leq-1 \text { is even. }
\end{array}\right.
$$

There is also a map of complexes $\ell: \operatorname{Cone}(e) \rightarrow \bar{T}[1]$ with

$\ell_{0}: P^{0} \oplus P_{g-1} \rightarrow P^{0}, \ell_{0}(x, y)=x,(x, y) \in P^{0} \oplus P_{g-1}$,

$\ell_{j}: P_{g-j} \oplus P^{j} \oplus P_{g-j-1} \rightarrow P^{j}, \ell_{j}(x, y, z)=y, \quad(x, y, z) \in P_{g-j} \oplus P^{j} \oplus$ $P_{g-j-1}, 1 \leq j \leq g-1$,

$\ell^{\prime}: P_{0} \oplus D \rightarrow D, \ell^{\prime}(x, y)=y,(x, y) \in P_{0} \oplus D$,

$$
\left\{\begin{array}{cl}
\ell_{j}=-i d_{P^{j}}, & \text { if } j \leq-1 \text { is odd } \\
\ell_{j}=i d_{P^{j}}, & \text { if } j \leq-1 \text { is even. }
\end{array}\right.
$$

We have

$$
\ell \circ \alpha=i d_{\bar{T}[1]} \text { and } \alpha \circ \ell \sim i d_{\operatorname{Cone}(e)} .
$$

(a chain homotopy between $\alpha \circ \ell$ and $i d_{\text {Cone }(e)}$ is given by the maps:

$\hbar_{0}: P_{0} \oplus D \rightarrow P_{1} \oplus P^{g-1} \oplus P_{0}, \hbar_{0}(x, y)=(0,0,-x)$,

$\hbar_{j}: P_{j} \oplus P^{g-j} \oplus P_{j-1} \rightarrow P_{j+1} \oplus P^{g-j-1} \oplus P_{j}, \hbar_{j}(x, y, z)=(0,0,-x), 1 \leq$ $j \leq g-2$,

$\left.\hbar_{g-1}: P_{g-1} \oplus P^{1} \oplus P_{g-2} \rightarrow P^{0} \oplus P_{g-1}, \hbar_{g-1}(x, y, z)=(0,-x)\right)$

So we have $\mathrm{H}^{n+1}\left(\operatorname{Hom}_{R}(\operatorname{Cone}(e), N)\right) \cong \mathrm{H}^{n+1}\left(\operatorname{Hom}_{R}(\bar{T}[1], N)\right)$, that is

$$
\widehat{\operatorname{Ext}}_{\mathcal{D P}}^{n}(M, N) \cong \widehat{\operatorname{Dext}}_{R}^{n}(M, N)
$$

for any $R$-module $N$ and for all $n \geq 1$.

The next Avramov-Martsinkovsky type exact sequence follows directly from Proposition 3.2(1), Theorem 5.2 and [13, Prop. 5.2].

Corollary 5.3. Let $M \in \widetilde{\mathcal{D P}}$ with $\operatorname{Dpd}_{R} M=g<\infty$. For each $R$-module $N$ there is an exact sequence

$$
\begin{aligned}
& 0 \longrightarrow \operatorname{Ext}_{\mathcal{D P}}^{1}(M, N) \longrightarrow \operatorname{Ext}_{R}^{1}(M, N) \longrightarrow \widehat{\operatorname{Dext}}_{R}^{1}(M, N) \longrightarrow \cdots \\
& \longrightarrow \operatorname{Ext}_{\mathcal{D P}}^{i}(M, N) \longrightarrow \operatorname{Ext}_{R}^{i}(M, N) \longrightarrow \widehat{\operatorname{Dext}}_{R}^{i}(M, N) \longrightarrow \cdots \\
& \longrightarrow \operatorname{Ext}_{R}^{g}(M, N) \longrightarrow \widehat{\operatorname{Dext}}_{R}^{g}(M, N) \longrightarrow
\end{aligned}
$$

Dually, we have the following: 
Corollary 5.4. Let $M$ be an $R$-module, $N \in \widetilde{\mathcal{D I}}$ with $\operatorname{Did}_{R} N=d<\infty$. Then there is an exact sequence

$$
\begin{aligned}
& 0 \longrightarrow \operatorname{Ext}_{\mathcal{D} \mathcal{I}}^{1}(M, N) \longrightarrow \operatorname{Ext}_{R}^{1}(M, N) \longrightarrow \overline{\operatorname{Dext}}_{R}^{1}(M, N) \longrightarrow \cdots \\
& \longrightarrow \operatorname{Ext}_{\mathcal{D} \mathcal{I}}^{i}(M, N) \longrightarrow \operatorname{Ext}_{R}^{i}(M, N) \longrightarrow \overline{\operatorname{Dext}}_{R}^{i}(M, N) \longrightarrow \cdots \\
& \longrightarrow \operatorname{Ext}_{R}^{d}(M, N) \longrightarrow \overline{\operatorname{Dext}}_{R}^{d}(M, N) \longrightarrow
\end{aligned}
$$

Since $\operatorname{Ext}_{\mathcal{D} \mathcal{P}}^{i}(M, N) \cong \operatorname{Ext}_{\mathcal{D I}}^{i}(M, N)$ for all $i \in \mathbb{Z}$ when $M \in \widetilde{\mathcal{D P}}$ and $N \in \widetilde{\mathcal{D I}}$ by Theorem 3.5, together with Definition 3.6, we have the following

Corollary 5.5. Let $M \in \widetilde{\mathcal{D P}}$ and $N \in \widetilde{\mathcal{D I}}$. Then there are exact sequences

and

$$
\begin{aligned}
0 \longrightarrow \operatorname{Dext}_{R}^{1}(M, N) \rightarrow \operatorname{Ext}_{R}^{1}(M, N) \rightarrow \widehat{\operatorname{Dext}}_{R}^{1}(M, N) \rightarrow \cdots \\
\longrightarrow \operatorname{Dext}_{R}^{i}(M, N) \longrightarrow \operatorname{Ext}_{R}^{i}(M, N) \longrightarrow \widehat{\operatorname{Dext}}_{R}^{i}(M, N) \longrightarrow \cdots
\end{aligned}
$$

$$
\begin{aligned}
0 \longrightarrow \operatorname{Dext}_{R}^{1}(M, N) \longrightarrow \operatorname{Ext}_{R}^{1}(M, N) \longrightarrow \overline{\operatorname{Dext}}_{R}^{1}(M, N) \longrightarrow \cdots \\
\longrightarrow \operatorname{Dext}_{R}^{i}(M, N) \longrightarrow \operatorname{Ext}_{R}^{i}(M, N) \longrightarrow \overline{\operatorname{Dext}}_{R}^{i}(M, N) \longrightarrow \cdots
\end{aligned}
$$

Over a Ding-Chen ring, Ren et al. proved that the generalized Tate cohomologies which they defined via Ding projective and Ding injective modules are isomorphic (see [13, Prop. 5.5]). However, as of the writing this paper, the author does not know whether the balance holds of the Tate cohomology relative to Ding projectives and Ding injectives in general. Anyhow, we have the following.

Theorem 5.6. If $R$ is a Ding-Chen ring, then for any $R$-modules $M \in \widetilde{\mathcal{D P}}$ and $N \in \widetilde{\mathcal{D I}}$ there is an Avramov-Martsinkovsky type exact sequence

or

$$
\begin{aligned}
0 \longrightarrow \operatorname{Dext}_{R}^{1}(M, N) \longrightarrow \operatorname{Ext}_{R}^{1}(M, N) \rightarrow \widehat{\operatorname{Dext}}_{R}^{1}(M, N) \longrightarrow \cdots \\
\longrightarrow \operatorname{Dext}_{R}^{i}(M, N) \longrightarrow \operatorname{Ext}_{R}^{i}(M, N) \longrightarrow \widehat{\operatorname{Dext}}_{R}^{i}(M, N) \longrightarrow \cdots
\end{aligned}
$$

$$
\begin{aligned}
0 \longrightarrow \operatorname{Dext}_{R}^{1}(M, N) \longrightarrow \operatorname{Ext}_{R}^{1}(M, N) \rightarrow \overline{\operatorname{Dext}}_{R}^{1}(M, N) \longrightarrow \cdots \\
\longrightarrow \operatorname{Dext}_{R}^{i}(M, N) \longrightarrow \operatorname{Ext}_{R}^{i}(M, N) \longrightarrow \overline{\operatorname{Dext}}_{R}^{i}(M, N) \longrightarrow \cdots
\end{aligned}
$$

At last, Theorem 5.2 and [13, Prop. 5.5] allow us to give an easy proof of the existence of a long exact sequences of Tate cohomology groups associated to any short exact sequences.

Proposition 5.7. Let $R$ be a Ding-Chen ring.

(1) Let $0 \rightarrow M^{\prime} \rightarrow M \rightarrow M^{\prime \prime} \rightarrow 0$ be an exact sequence of $R$-modules. For any $R$-module $N \in \widetilde{\mathcal{D I}}$ there exist a long exact sequence of Tate cohomolgy 
groups

$$
\begin{aligned}
\cdots & \rightarrow \widehat{\operatorname{Dext}}_{R}^{n-1}\left(M^{\prime \prime}, N\right) \longrightarrow \widehat{\operatorname{Dext}}_{R}^{n-1}(M, N) \rightarrow{\widehat{\operatorname{Dext}_{R}}}_{R}^{n-1}\left(M^{\prime}, N\right) \\
& \longrightarrow \widehat{\operatorname{Dext}}_{R}^{n}\left(M^{\prime \prime}, N\right) \longrightarrow \cdots .
\end{aligned}
$$

(2) Let $0 \rightarrow N^{\prime} \rightarrow N \rightarrow N^{\prime \prime} \rightarrow 0$ be an exact sequence of $R$-modules. For each $R$-module $M \in \widetilde{\mathcal{D P}}$, there exists a long exact sequence of Tate cohomology groups

$$
\begin{aligned}
\cdots & \longrightarrow \overline{\operatorname{Dext}}_{R}^{n-1}\left(M, N^{\prime}\right) \longrightarrow \overline{\operatorname{Dext}}_{R}^{n-1}(M, N) \rightarrow \overline{\operatorname{Dext}}_{R}^{n-1}\left(M, N^{\prime \prime}\right) \\
& \longrightarrow \overline{\operatorname{Dext}}_{R}^{n}\left(M, N^{\prime}\right) \longrightarrow \cdots .
\end{aligned}
$$

Proof. (1) Let $N \stackrel{\lambda}{\longrightarrow} I \stackrel{\nu}{\longrightarrow} S$ be a Tate $\mathcal{F} \mathcal{I}$-coresolution of $N$. Then, by [13, Prop. 5.5], $\widehat{\operatorname{Dext}}_{R}^{n}(M, N) \cong \overline{\operatorname{Dext}}_{R}^{n}(M, N)=\mathrm{H}^{-n}\left(\operatorname{Hom}_{R}(M, S)\right)$ for any $R$ module $M$ and any $n \in \mathbb{Z}$. Since $0 \rightarrow M^{\prime} \rightarrow M \rightarrow M^{\prime \prime} \rightarrow 0$ is exact and each term of $S$ is an injective module, we have an exact sequence of complexes:

$$
0 \longrightarrow \operatorname{Hom}_{R}\left(M^{\prime \prime}, S\right) \longrightarrow \operatorname{Hom}_{R}(M, S) \longrightarrow \operatorname{Hom}_{R}\left(M^{\prime}, S\right) \longrightarrow 0 .
$$

Its associated cohomology exact sequence is the desired long exact sequence.

(2) The proof is dual to (1).

Acknowledgements. This research was supported by National Natural Science Foundation of China (No. 11261050, 11301240, 11401475) and by NWNUlkqn-12-17. The authors would like to thank the referees for the very helpful suggestions and corrections that have improved this article.

\section{References}

[1] L. L. Avramov and H.-B. Foxby, Homological dimension of unbounded complexes, J. Pure Appl. Algebra 71 (1991), no. 2-3, 129-155.

[2] L. L. Avramov and A. Martsinkovsky, Absolute, relative, and Tate cohomology of modules of finite Gorenstein dimension, Proc. London Math. Soc. 85 (2002), no. 2, 393-440.

[3] N. Q. Ding, Y. L. Li, and L. X. Mao, Strongly Gorenstein flat modules, J. Aust. Math. Soc. 86 (2009), no. 3, 323-338.

[4] E. E. Enochs and O. M. G. Jenda, Resolutions by Gorenstein injective and projective modules and modules of finite injective dimension over Gorenstein rings, Comm. Algebra 23 (1995), no. 3, 869-877.

[5] L Relative homological algebra, De Gruyter Expositions in Mathematics no. 30, Walter De Gruyter, New York, 2000.

[6] J. Gillespie, Model structures on modules over Ding-Chen rings, Homology Homotopy Appl. 12 (2010), no. 1, 61-73.

[7] R. Hartshorne, Algebraic Geometry, Graduate Texts in Mathematics 52, SpringerVerlag, New York-Heidelberg, 1977.

[8] H. Holm, Gorenstein derived functors, Proc. Amer. Math. Soc. 132 (2004), no. 7, 19131923.

[9] A. Iacob, Generalized Tate cohomology, Tsukuba J. Math. 29 (2005), no. 2, 389-404. 
[10] N. Mahdou and M. Tamekkante, Strongly Gorenstein flat modules and dimensions, Chin. Ann. Math. Ser. B 32 (2011), no. 4, 533-548.

[11] L. X. Mao and N. Q. Ding, Gorenstein FP-injective and Gorenstein flat modules, J. Algebra Appl. 7 (2008), no. 4, 491-506.

[12] K. Pinzon, Absolutely pure covers, Comm. Algebra 36 (2008), 2186-2194.

[13] W. Ren, Z. K. Liu, and G. Yang, Derived categories with respect to Ding modules, J. Algebra Appl. 12 (2013), no. 6, 1350021, 14 pp.

[14] O. Veliche, Gorenstein projective dimension for complexes, Trans. Amer. Math. Soc. 358 (2006), no. 3, 1257-1283.

[15] Z. P. Wang, Reseaches of relative homological properties in the category of complexes, Ph.D. thesis, Northwest Normal University, China, 2010.

[16] C. H. Yang, Strongly Gorenstein flat and Gorenstein FP-injective modules, Turkish J. Math. 37 (2013), no. 2, 218-230.

[17] G. Yang, Homological properties of modules over Ding-Chen rings, J. Korean Math. Soc. 49 (2012), no. 1, 31-47.

[18] G. Yang, Z. K. Liu, and L. Liang, Ding projective and Ding injective modules, Algebra Colloq. 20 (2013), no. 4, 601-612.

Department of Mathematics

NORTHWEST NORMAL UNIVERSITY

LANZHOU 730070, P. R. ChINA

E-mail address: zhangcx@nwnu.edu.cn 